\title{
MULTIDISCIPLINARY MODELLING, ANALYSIS AND OPTIMIZATION FOR AIRCRAFT AND SYSTEM LEVEL DESIGN AND VALIDATION
}

\author{
W.J. VANKAN ${ }^{1}$, W.F. LAMMEN' ${ }^{\text {, E.H. BAALBERGEN }}{ }^{1}$
}

${ }^{1}$ Royal Netherlands Aerospace Centre NLR

Aerospace Vehicles Division, Collaborative Engineering Systems Department Anthony Fokkerweg 2, 1059 CM Amsterdam, The Netherlands

jos.vankan@nlr.nl, www.nlr.nl

Key words: MDO, Collaborative engineering, BRICS, MULTIFIT, SMR.

\begin{abstract}
Against the background of the big environmental and societal challenges as formulated for example in Flightpath 2050, current developments in aircraft design are aiming at further emission reduction through integrated, unconventional propulsion, systems and airframe innovations. This requires the further integration of methods for multidisciplinary modelling, analysis and optimization for aircraft design, but also for propulsion and system level designs. Moreover, experimental validation of the methods and physical testing of critical unconventional propulsion and system designs are prerequisites for industrially relevant development processes. This paper presents some key technologies for computationally efficient collaborative MDO (multidisciplinary design and optimization) frameworks for multidisciplinary design and validation of advanced aeronautic products like aircraft and propulsion systems.
\end{abstract}

\section{INTRODUCTION}

Aircraft design is inherently a multidisciplinary process: many different disciplines are involved and each discipline plays a key role in the aircraft design process.

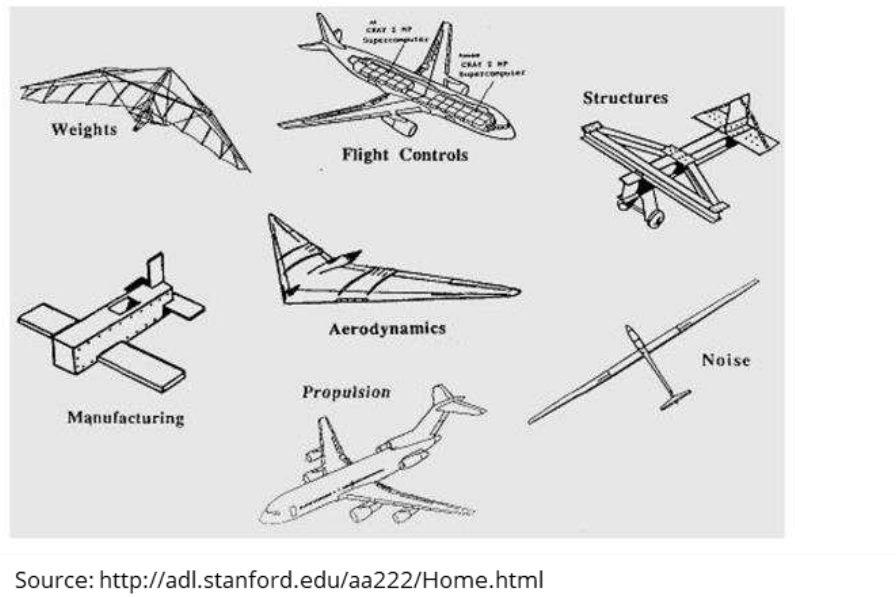

Figure 1: illustration of the multiple disciplines involved in the aircraft design process. 
In contrast with what is shown in the picture above, a successful aircraft design requires that each of these different disciplines must contribute to the overall design objectives. Therefore these different disciplines must be well balanced and must be integrated into one coherent multidisciplinary design process. Optimization methods then are typically used to steer this multidisciplinary design process in the right direction.

Multidisciplinary Modelling \& Analysis is of key importance, not only in the aircraft design process, but also for System Level Design and Validation. As an example we can look at the Clean Sky 2 (CS2) project PROPMAT [1], where NLR is working at Future Propulsion System Maturation.

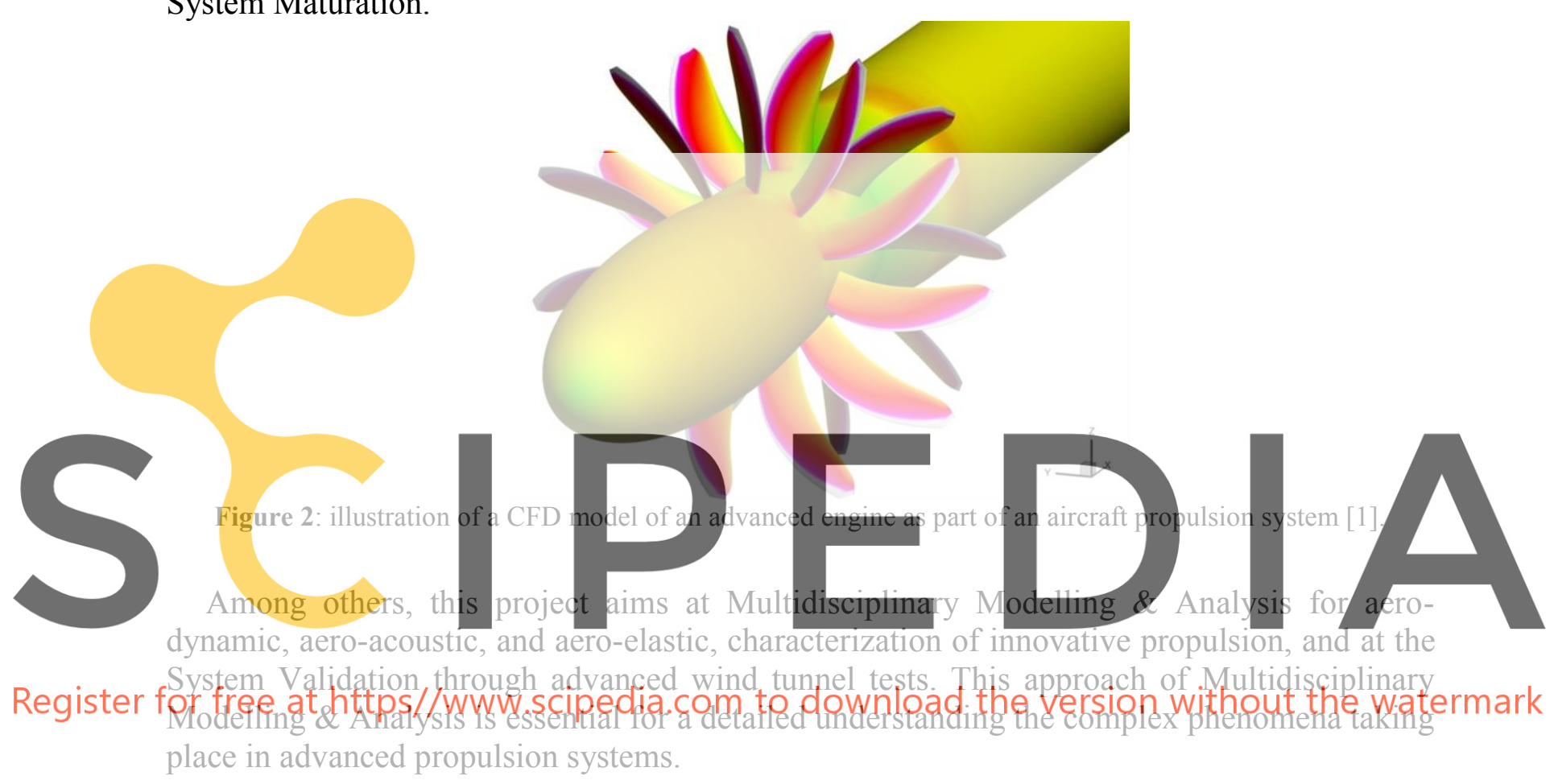

Besides many different disciplines, also many different people are typically involved in the aircraft design process.

These different people may be from different groups or departments within one organization. But quite commonly people from different organizations are involved in the aircraft design process. These different organizations are often international partners, each playing their role in a supply chain collaboration. In such a collaboration it is essential that information from each of the partners can be efficiently shared and exchange $d$ with the other partners. In the Horizon-2020 research project AGILE [2] also a number of different international partners collaborate in various aircraft design processes. 


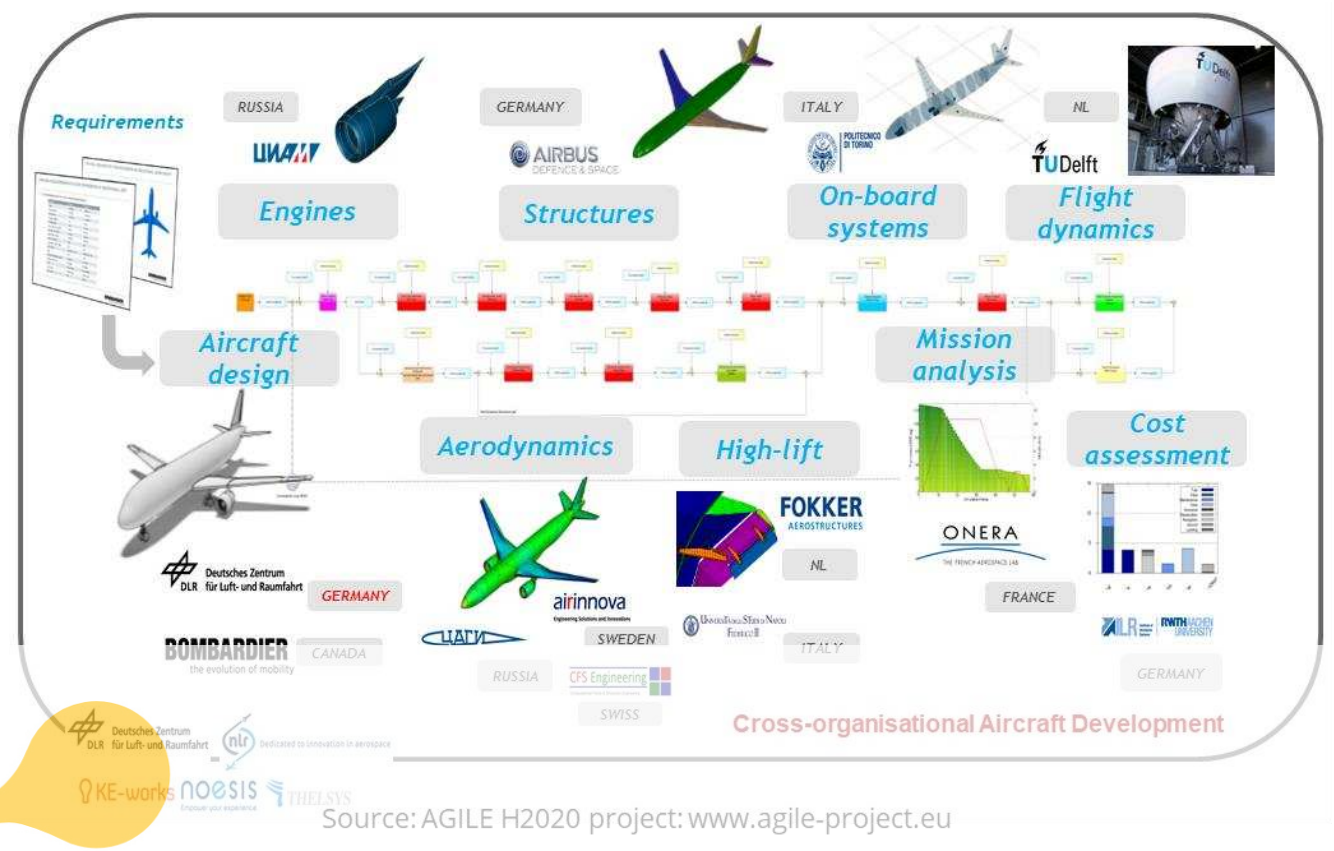

Figure 3: Illustration of the different roles that partners play in the various aircraft design processes, as executed by the different international partners that collaborate on various design use-cases in the Horizon-2020 research project AGILE.
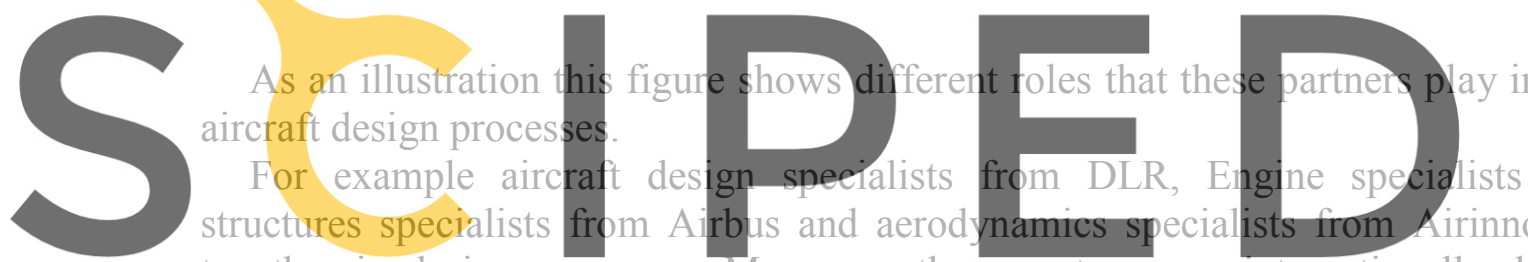

together in design use cases. Moreover, these partners are

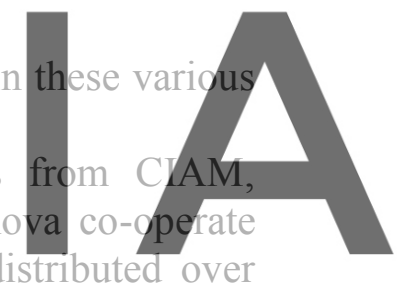
Register formany, Russia and Sweden, which limits the possibilities for physically co-located

So, besides the multi-disciplinary aspects of MDO, also the collaborative aspects play an important role in MDO. The Agile project has been looking, among others, at these collaborative aspects of MDO. This paper presents some key technologies developed in AGILE for computationally efficient collaborative MDO frameworks for multidisciplinary design and validation of advanced aeronautic products like aircraft and propulsion systems. Besides this general introduction on collaborative MDO, it will present some technology developments for aircraft design and collaborative MDO. The role of surrogate models in design analysis will be explained and an example application in a use case study will be briefly shown.

\section{COLLABORATIVE ENVIRONMENTS AND MDO}

Let us first take a step back, and look a bit more globally at MDO. MDO has a long history in aerospace. Already in the 1980's multidisciplinary modelling and simulation for aircraft design was combined with optimization methodologies. 
Towards the next Generation MDO

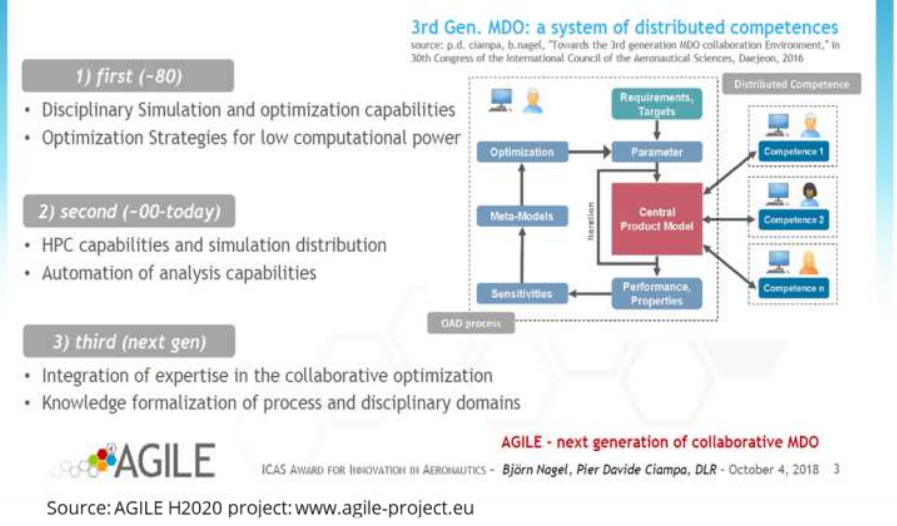

Figure 4: Illustration of the different generations of MDO systems from the recent decades.[2]

For example structural analyses with finite element models were combined with aerodynamic loads analyses and used in automated sizing procedures. These approaches can be considered as the first generation of MDO systems. In later decades, more and more high performance computation capabilities came available that were used in more advanced models and distributed simulations.

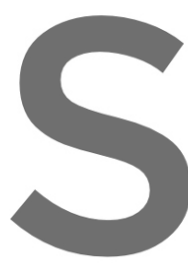

These approaches example, the NLR e-C (HPC) facilities and available to external
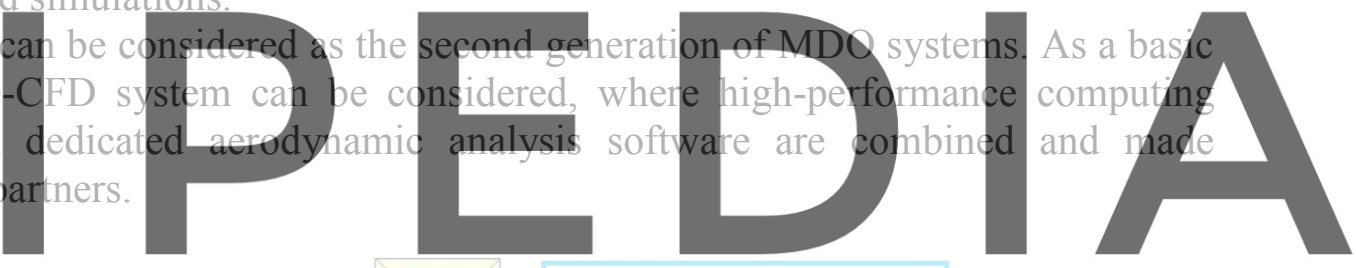

Register for free at https//www.scipedia.com to download the version without the watermark

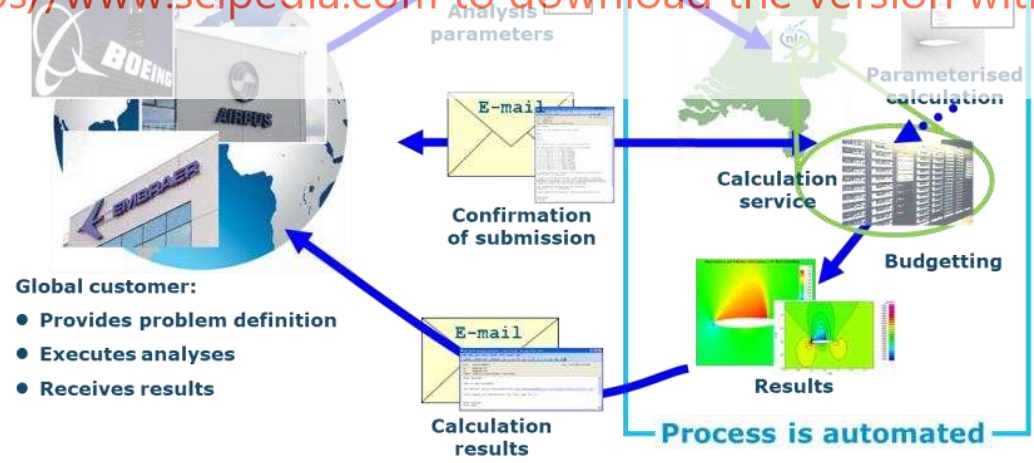

Figure 5: Illustration of the NLR E-CFD system: Remote access to models \& methods for complex aircraft component calculations.

The e-CFD system was originally set up at NLR as an analysis service for our customers. E-CFD can be seen as a basic type of cloud computing. But the specific aspects of it are the following:

- The specific aerodynamic analyses with the dedicated in-house analysis software and 
HPC facilities are set up by NLR specialists in close co-operation with the customer;

- The customer can very easily execute and operate these analysis via very basic emailbased communication.

This has several advantages:

- The customer can very efficiently exploit the specific expertise of the NLR specialists on the analysis software in combination with the HPC facilities;

- The access and control of the analyses is very thin in terms of data, and networksecurity-independent because of the use of the very generic email protocol.

The figure illustrates the process for a very basic example analysis with the E-CFD system:

1. First, the global customer and NLR co-operate on the exact problem definition.

2. NLR specialists take care of the adequate parametric implementation of the problem on NLR's calculation service.

3. The customer initiates the analysis by simply sending an email to a designated email address(ecfd@nlr.nl)

4. The customer receives an email with confirmation of submission, including submission details and for example budget and cost information

5. The analysis is executed and results are stored on a data server

6. A link for retrieval of the calculation results is sent to customer by email. The complete analysis process and data exchange are automated.
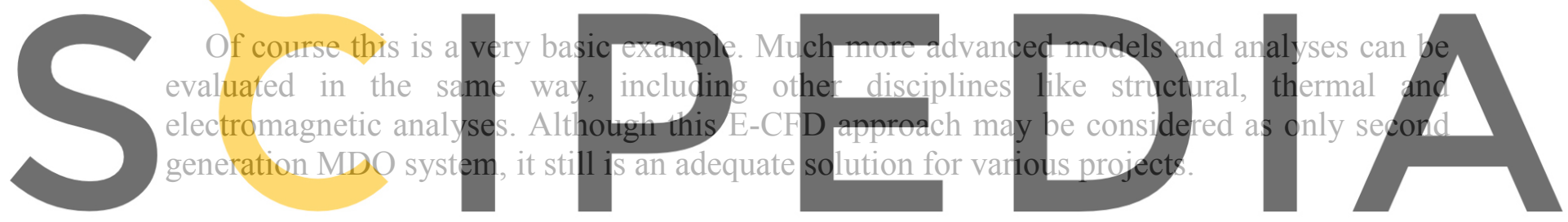

In more recent and ongoing projects, like the Horizon-2020 projects AGILE and

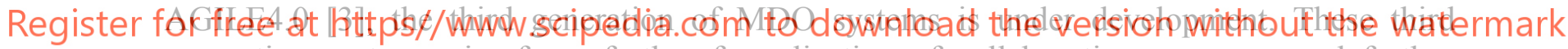
generation systems aim for a further formalization of collaborative processes and further integration of collaborative technologies. Such more advanced collaborative technologies are also being developed at NLR, among others a software system called BRICS [4].

BRICS is an advanced collaborative technology under development at NLR. BRICS supports cross-organization collaborative design workflows. Of course these workflows can deal with aircraft design, or with any other complex design process. BRICS can be seen as a generalization of e-CFD: it applies some parts of the e-CFD bi-lateral data exchange protocols to multi-lateral collaborative workflows. BRICS can be explained as follows, where it is illustrated by a design use case from the AGILE project, following some key process steps:

1. Given a typical collaborative a/c design process, which includes various analysis activities distributed over different partners.

2. The aim is to integrate this design process into a single collaborative distributed Workflow. RCE, CMDOWS and KE-chain are shown here as workflow implementation tools. What is also needed, besides these workflow implementation tools, is a versatile data exchange and execution protocol that can efficiently interconnect these distributed analysis activities.

3. BRICS provides this protocol plus supportive middleware to turn this distributed set of 
analysis-tools and expert-workflows into an integrated cross-organization collaborative workflow.

4. BRICS is available as plug-in for RCE. But BRICS is also available in other forms, like Java packages and scripts that can be integrated in commercial software packages.

5. Establishing and running cross-organization MDO workflows comes with specific challenges, such as:

a. Access limitations due to Prevailing security constraints or IPR protection

b. Collaborating Partners who want to keep full control of their own resources

c. Security measures and network restrictions that impede smooth execution of workflows across organizations

d. Heterogeneity of workflows, of analysis and optimization tools and of ways-ofworking

e. Dynamic IT environments that impose changes over time.

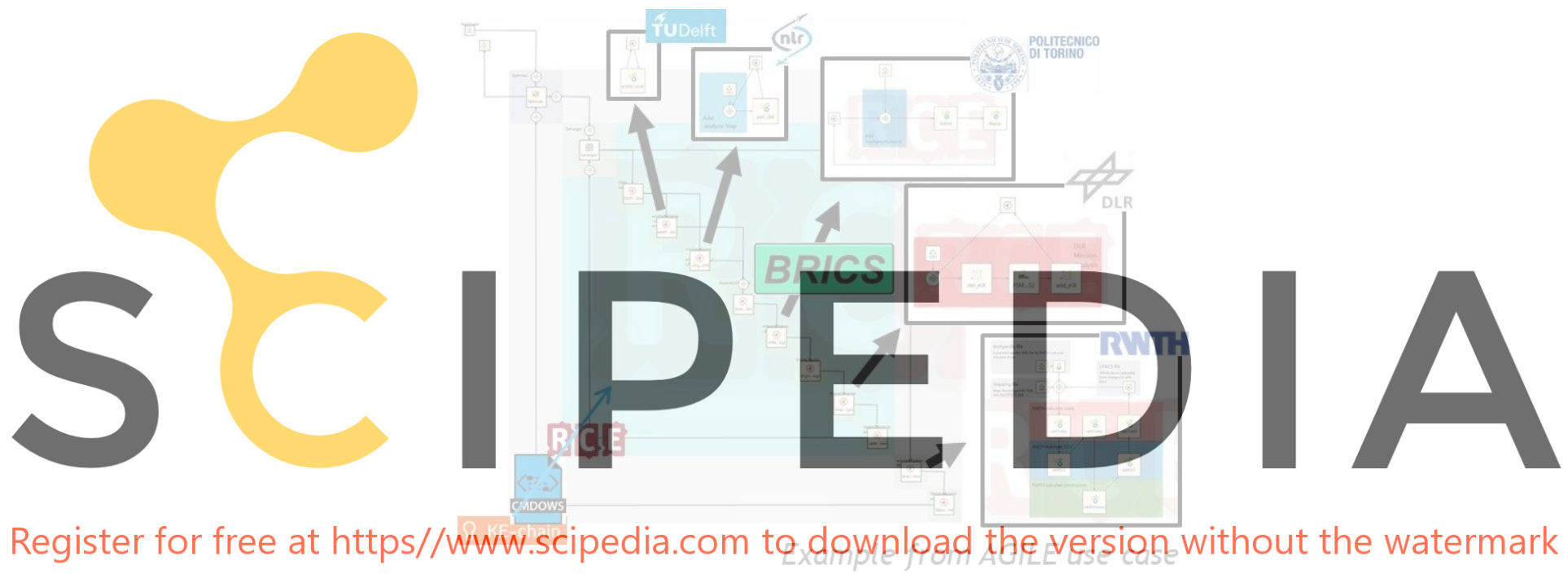

Source: AGILE H2020 project: www.agile-project.eu

Figure 6: Illustration of the NLR BRICS system: establishing cross-organization collaborative aircraft design workflows.

The difference between a basic local workflow process and a multi-site collaborative workflow on different computers in different organizations, potentially in different countries, involves several challenges. These challenges are related to the heterogeneity among organizations, people and their ways of working, and by the security constraints that serve to protect assets and intellectual property (IP). As a result, collaborating engineers from different organizations generally have to deal with barriers such as compliance with information and knowledge sharing rules, the obligation to exchange data securely, technical security measures (incl. firewalls, proxy servers), and heterogeneity among working procedures, tools and data formats. These clear and explicit barriers exist in addition to more implicit barriers and issues such as export control regulations, cultural differences, personal and organizational interests and lack of an overarching organizational management. 
Of course, BRICS does not automatically deal with all these challenges, but it provides the means to solve the related problems in a structured way.

\section{SURROGATE MODELS IN MDO}

Besides the data protocols and middleware, also surrogate models play an important role in collaborative MDO studies during early aircraft design phases. Surrogate models are valuable to support in collaborative aircraft design processes. For example, Suppliers of aircraft parts and systems can collaborate with the aircraft integrator by providing surrogate models without exposing their IP, in contrast to sharing high fidelity models which contain much more of the company's proprietary knowledge.

\subsection{MultiFit}

A surrogate model is a simplified model, usually based on analytical formulas, that is tuned for a certain target by data fitting. A surrogate model can be used to replace a complex model, like an aerodynamic analysis model, or even a design analysis workflow. A surrogate model is computationally efficient, which is useful for replacing complex analyses, in particular when applied in highly iterative evaluations like in global optimization. NLR has a long

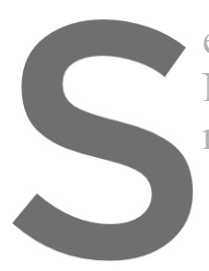
experience in developin

NLR has developed modelling \& Optimizat
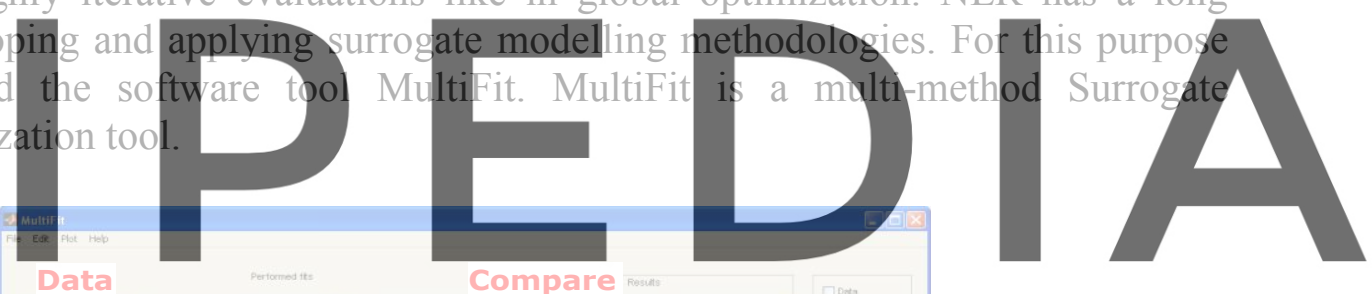

Register for free at https//www.scipedia.com to download the versign without the watermark

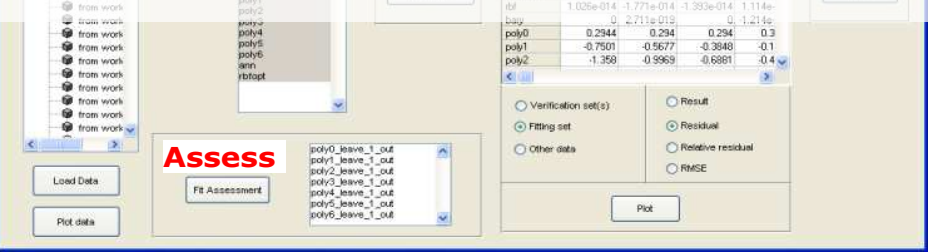

Figure 7: Illustration of the NLR MultiFit tool for multi-dimensional data fitting with multiple algorithms and integrated data analysis and fit accuracy assessment.

MultiFit has been partly developed and deployed in recent R\&D projects, among others:

- H2020-AGILE on collaborative MDO

- EU-FP7 MAAXIMUS on Composite fuselage optimization

- $\quad$ EU-FP7 TOICA on Thermal Aircraft modelling

MultiFit supports Multi-dimensional data fitting with multiple algorithms, like polynomials, splines, and neural nets. MultiFit supports Data analysis and fit accuracy 
assessment algorithms with statistical measures like r-squared and cross-validation etc. MultiFit is developed in Matlab [5] and builds on Matlab functionality. MultiFit can be combined with some in-house implementations for Global Optimization, like $\varepsilon$-MOEA and $\varepsilon$ NSGA. Of course, also more general optimization algorithms can be used in Matlab for constrained and unconstrained optimization. These tools have been applied extensively in different projects and use cases.

\subsection{Surrogate Model Repository - SMR}

In collaborative MDO workflows, surrogate models must be carefully applied. For example, the bounds of the allowed input space of the surrogate model need to be clearly specified (e.g. to avoid extrapolation). Furthermore the prediction accuracy of the outputs of the surrogate model must be specified, so that the user has a clear idea of its applicability, quality and limitations. Many surrogate models of different types may be used in a collaborative aircraft design project, e.g. to support efficient optimization and partner collaboration. The question then rises how to manage, i.e., document, register, deploy and share these surrogate models? Various aspects need to be taken into account in answering this question, such as:

\section{IP issues: can the surrogate model be shared or does it still contain intellectual property that is considered confidential information?}
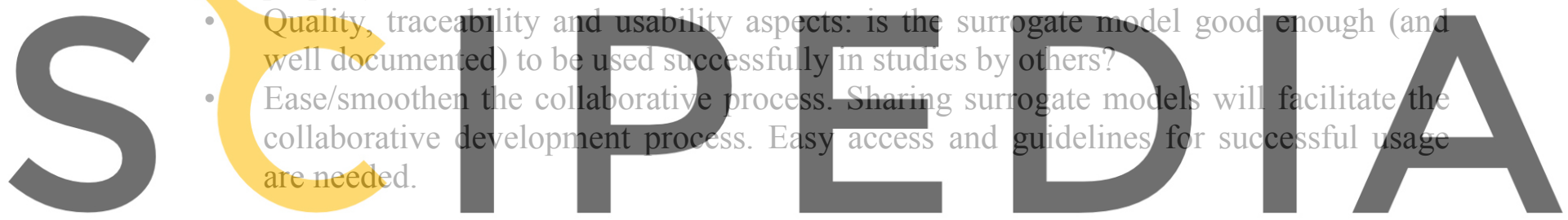

Based on these aspects, a Surrogate Model Repository (SMR) has been developed at NLR Register fon. free at https//www.scipedia.com to download the version without the watermark

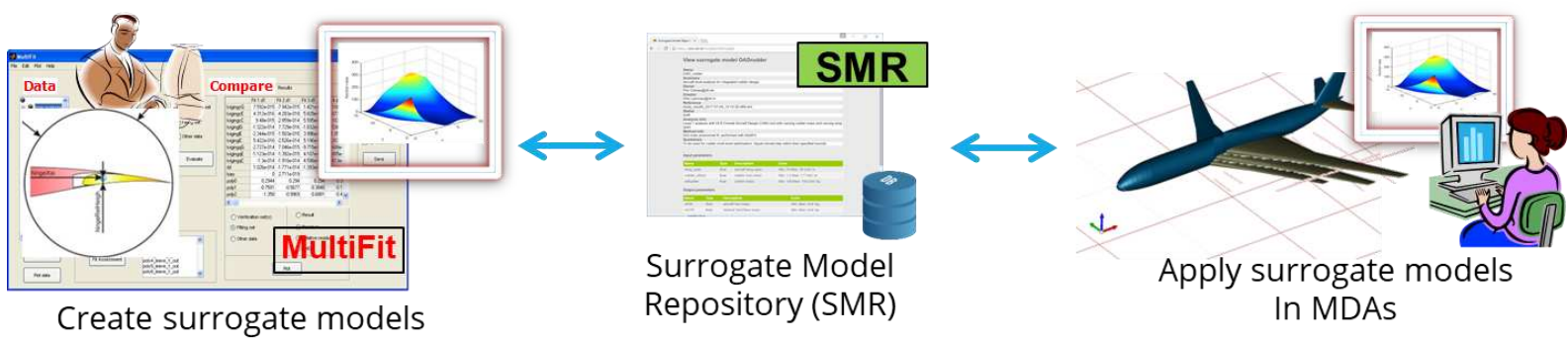

Figure 8: Illustration of the NLR Surrogate Model Repository (SMR): central broker for registration, storage, deployment, sharing, and usage of surrogate models in collaborative multidisciplinary analysis.

The SMR is defined as central broker for registration, storage, deployment, sharing, and usage of surrogate models so that these may be shared and reused in collaborations in a managed way. The SMR stores meta-information with a surrogate model. The metainformation contains certain properties that can be assigned to a surrogate model. The meta- 
information also facilitates easy usage of surrogate models e.g. by being searchable and crossreferencing the source dataset, fitting method, IP owner, etc. The meta-information includes the following items:

- General Information: creation date, creator (e.g. name, company, email), owner (may be different from the creator), operator (envisaged user of the surrogate model), version, status (draft/final);

- Description: guidelines of usage, reference to the data set from which the surrogate model (SM) was derived, description of original (high-fidelity) model(s) or analysis used for creating the data set (including version and owner) as well as fitting method (including related surrogate modelling tools that have been applied to create the SM);

- Input and Output variables: name, description, physical unit, type (e.g. float), bounds (for input only);

- Verification: verifier (e.g. name, company, email), verification date, method, result: output prediction error metric and value per output variable;

- Execution information: downloadable executable or "available as-a-service" (also provided online through SMR).

With respect to the sharing of surrogate models different cases are considered in SMR:

Full share: Share all of the compiled binary code of a SM to support its use by others.

In this case the complete SM implementation is uploaded to the SMR with meta-

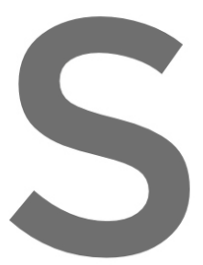

information describing its usage. The SM is used by downloading its code and running
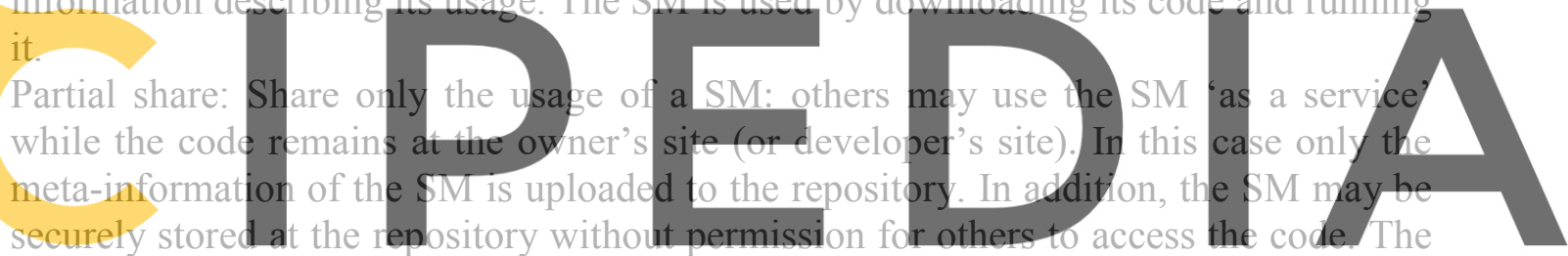

SMR provides a user interface to directly use this SM by calling the remote service.

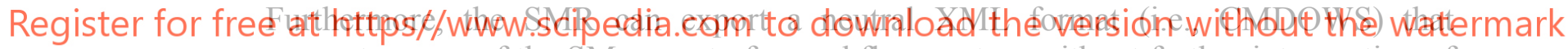
supports usage of the SM as part of a workflow system without further intervention of the SMR.

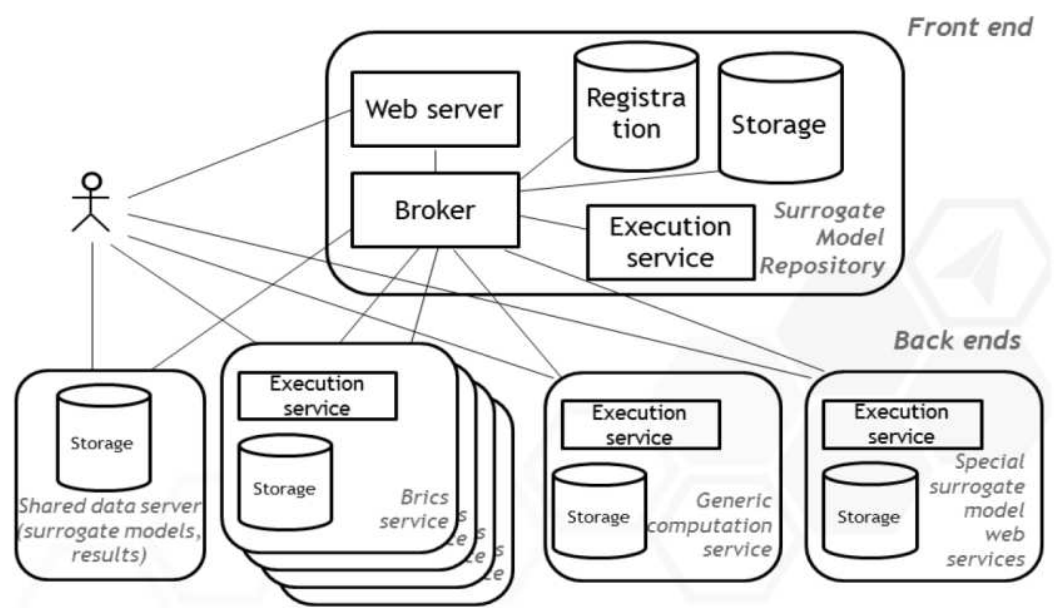

Figure 9: Illustration of the NLR SMR implementation architecture [4]. 
The implementation of SMR is modular in order to be flexible to support integration of different services. This modular architecture is illustrated by the schema on the right. A separation is made between the Front End and the various Back Ends services of SMR. The Front End provides the main functionality of the actual SMR implementation, including the web interface, web server, registration and broker functionality. The various Back Ends provide the storage and execution capabilities of SMR. The back ends may be located remotely with respect to the front end. The back ends provide the services for storage and remote execution of surrogate models.

\section{APPLICATION - RUDDER DESIGN USE CASE}

\section{The BRICS and SMR tools have been used in the AGILE project use case on collaborative} aircraft rudder multilevel optimization using surrogate models. The use case involves the multilevel optimization (MLO) of an aircraft rudder. In this collaborative design case the choices in the aircraft design may impact the rudder design, e.g. through the rudder planform sizes that follow from the aircraft design and by the applicable rudder forces that result from the design load cases.
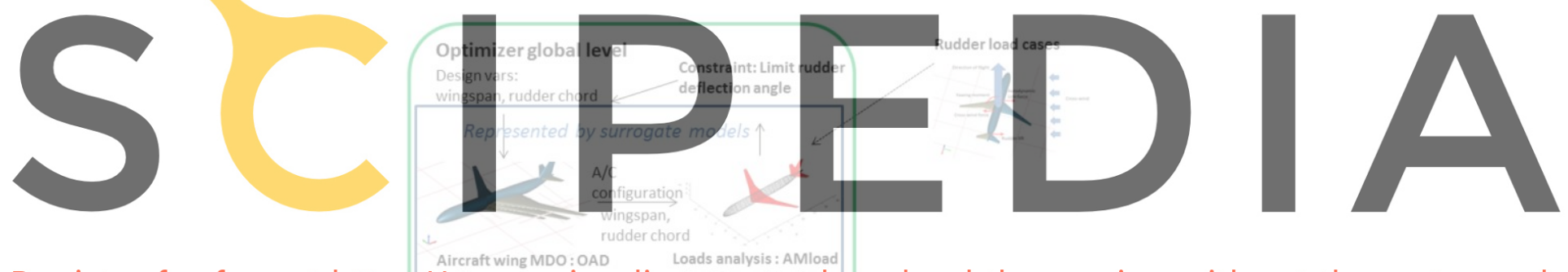

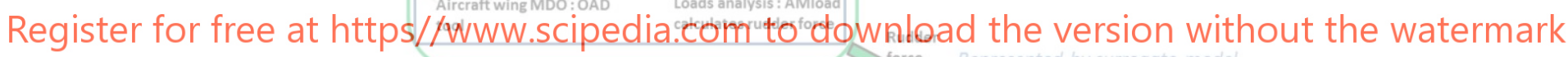

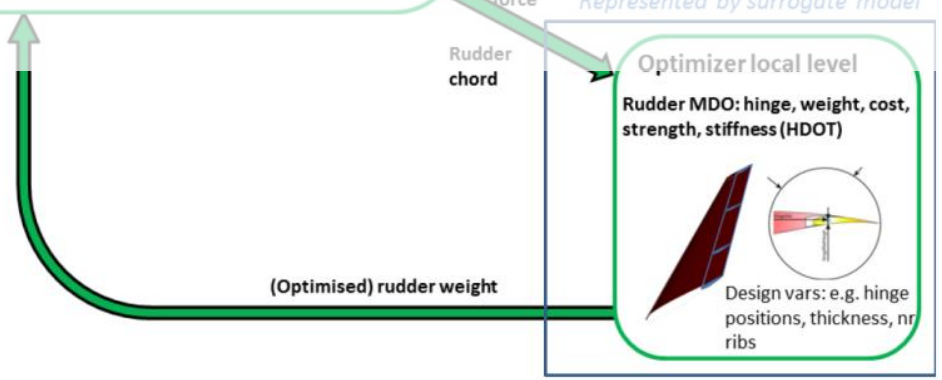

Figure 10: Illustration of the AGILE project use case on collaborative aircraft rudder multilevel optimization using surrogate models [6].

In this use case, on the optimizer global level, two surrogate models have been developed that represent the aircraft level design analysis in the context of aircraft rudder MLO. The first surrogate model has been derived from data calculated with an Overall Aircraft Design (OAD) analysis capability provided by DLR. The second surrogate model has been derived 
from simulations with a tool for aircraft load calculation provided by NLR: AMLoad. The surrogate models are shared and deployed as-a-service through the SMR. In this way other partners such as the rudder supplier can use the surrogate models to test potential impact of changes in aircraft design on the rudder design and anticipate on that.

On the optimizer local level, another surrogate model has been developed that represents the rudder design. A surrogate model has been derived that predicts optimal rudder mass as a function of specified rudder sizes and loads. This surrogate model is also shared and deployed as-a-service through the SMR.

All these surrogate models are shared and deployed as-a-service through the SMR.

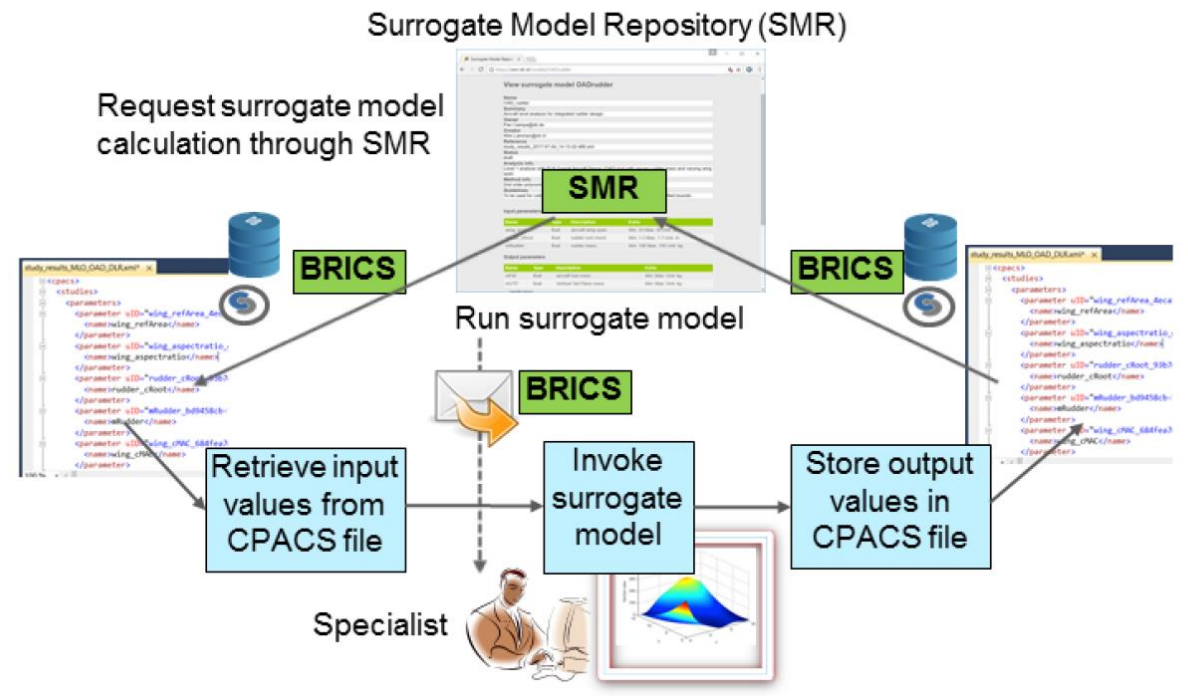

Figure 11: Schematic view on the implementation of the rudder design use case [6].

A schematic view on the implementation of the rudder design use case is shown in the figure. In this implementation, the SMR is used to retrieve the information of a surrogate model, in this case the OAD surrogate model, and to request an online calculation with the surrogate model. This online calculation is provided as-a-service, using the same Brics technology as was also applied in the context of cross-organization workflows

The input and output data of the surrogate model are stored in CPACS format, using the specific CPACS 'designStudy' object. As such a list of several combinations of design parameter values (the inputs) can be processed by the surrogate model in one step. The corresponding CPACS files can be both loaded and generated using the SMR. The user can specify the input values manually via the SMR user interface or select and load a specific CPACS file to provide a set of input values. The results of the calculations with the surrogate model (the outputs) are presented to the SMR user interface.

This paper can only give a global illustration of the application of the BRICS and SMR technologies in a collaborative aircraft design use case. The full details of this use case cannot be presented here, but are extensively reported in a previous paper [6]. 


\section{CONCLUSIONS}

In this paper it has been shown that efficient collaboration is of key importance in the complex multi-disciplinary aircraft design process But such multi-partner collaboration in complex multi-disciplinary design processes is not trivial; barriers exist. Knowledge sharing in collaboration and streamlined integration in the virtual enterprise are important aspects in 3rd generation MDO systems and can be achieved by:

- provision of tools "as a service" (through BRICS) for efficient and secure integration of engineering workflows at multiple partners

- $\quad$ sharing surrogate models in a central repository (SMR)

The technologies, presented in this paper, that have been developed for both these aspects:

- are non-intrusive for IT domains and can cross company borders while complying with security policies

- have been implemented and tested in the AGILE project and have the potential to be scaled up to large collaborative aircraft design projects.

\section{Acknowledgements}

The research presented in this paper has been performed in the framework of the AGILE project (Aircraft 3rd Generation MDO for Innovative Collaboration of Heterogeneous Teams of Experts) and has received funding from the European Union Horizon 2020 Programme (H2020-MG-2014-2015) under grant agreement no. 636202.

PROPMAT has received funding from the Clean Sky 2 Joint Undertaking under the European Union's Horizon 2020 research and innovation programme under grant agreement No 680954.

\section{REFERENCES}

[1] PROPMAT project, https://cordis.europa.eu/project/id/680954.

[2] AGILE project, https://www.agile-project.eu/.

[3] AGILE4.0 project, https://www.agile4.eu/ .

[4] Baalbergen, E.H., Moerland, E., Lammen, W.F., Ciampa, P.D., Methods Supporting the Efficient Collaborative Design of Future Aircraft, Aerospace Europe CEAS 2017 Conference, Bucharest, Romania, October 16-20, 2017. NLR-TP-2017-338.

[5] Mathworks, https://nl.mathworks.com/products/matlab.html.

[6] A.J. de Wit, W.F. Lammen, W.J. Vankan, H. Timmermans, T. van der Laan, P.D. Ciampa, Aircraft rudder optimization - A multi-level and knowledge-enabled approach, Progress in Aerospace Sciences 119, 2020. 